\title{
Dissociation in the effects of neonatal maternal separations on maternal care and the offspring's HPA and fear responses in rats
}

\author{
Simone Macrí, ${ }^{1}$ Georgia J. Mason ${ }^{2}$ and Hanno Würbel ${ }^{3}$ \\ ${ }_{1}^{1}$ Institute of Animal Sciences, Swiss Federal Institute of Technology (ETH), Schorenstrasse 16, 8603 Schwerzenbach, Switzerland \\ ${ }^{2}$ Department of Zoology, University of Oxford, South Parks Road, Oxford, OX1 3PS, UK \\ ${ }^{3}$ Institute of Veterinary Physiology, Justus-Liebig-University of Giessen, Frankfurter Strasse 104, 35392 Giessen, Germany
}

Keywords: early handling, fear, maternal care, maternal separation, stress

\begin{abstract}
The development of the hypothalamic-pituitary-adrenal (HPA) response to stress is influenced by the early mother-infant relationship. In rats, early handling (brief daily mother-offspring separations) attenuates the adult offspring's HPA and fear responses compared to both nonhandling (no separations) and maternal separation (prolonged daily separations). It has been proposed that variation in the amount of maternal care mediates these effects of neonatal manipulations on the adult offspring's stress and fear responses. Here we tested this hypothesis by assessing maternal care and the adult offspring's HPA and fear responses in Lister hooded rats which were subjected to either early handling $(E H)$ or maternal separation (MS) from postnatal day 1-13, or were left completely undisturbed (nonhandled, $\mathrm{NH}$ ) throughout this period. Both EH and MS induced a more active nursing style and elevated levels of maternal care compared to NH. Total levels of maternal care were indistinguishable between EH and MS, but diurnal distribution differed. MS dams showed elevated levels of maternal care following the 4-h separation period, thereby fully compensating for the amount of maternal care provided by EH dams during the time MS dams were separated from their pups. However, while EH resulted in reduced HPA and fear responses in the adult offspring compared to NH, MS and NH offspring did not differ. Our findings therefore demonstrate dissociation in the effects of EH and MS on maternal care and on the stress and fear responses in the offspring. This indicates that maternal care cannot be the sole mediator of these effects.
\end{abstract}

\section{Introduction}

In both human and nonhuman mammals, the development of the hypothalamic-pituitary-adrenocortical (HPA) system and related defensive systems is influenced by the infant-mother relationship (Meaney, 2001; Pryce \& Feldon, 2003). In rats, early handling (a 3-15-min daily separation of pups and dam) consistently reduces adult HPA responses to stress (Levine, 1957, 1962; Levine et al., 1967; Ader \& Grota, 1969; Meaney et al., 1989; Viau et al., 1993; Bhatnagar et al., 1995; Liu et al., 2000) and behavioural fearfulness (Levine, 1957, 1962; Denenberg, 1964; Bodnoff et al., 1987; Caldji et al., 2000a) compared to nonhandled controls.

In contrast, it has been proposed that postnatal maternal separation (a 3-6-h daily separation of pups and dam) induces precisely the opposite effect to early handling, i.e. to increase adult HPA responses to stress and behavioural fearfulness compared to nonhandled controls (Francis \& Meaney, 1999; Meaney, 2001). However, this manipulation has produced far less consistent results. In some studies, maternal separation was indeed found to increase HPA responses (e.g. Biagini et al., 1998; McCormick et al., 2002) or measures of fearfulness (e.g. Plotsky \& Meaney, 1993) compared to nonhandling but, in others, no significant effects on HPA responses (e.g. Plotsky \& Meaney, 1993;

Correspondence: Professor Dr Hanno Würbel, as above.

E-mail: hanno.wuerbel@vetmed.uni-giessen.de

Received 9 February 2004, revised 22 April 2004, accepted 7 June 2004
Liu et al., 2000) or fearfulness (e.g. Hilakivi-Clarke et al., 1991; Caldji et al., 2000b) were detected, and in yet others even the opposite result emerged (see McIntosh et al., 1999 for reduced fearfulness and Ogawa et al., 1994 for reduced HPA responsiveness).

Early handling induces elevated levels of maternal care compared to nonhandling (Liu et al., 1997). Furthermore, spontaneous high levels of active maternal care in nonhandled dams mimics the effects of early handling in terms of attenuated HPA and fear responses in the offspring (Liu et al., 1997; Caldji et al., 1998). In line with the maternal mediation hypothesis earlier formulated by Smotherman \& Bell (1980) it was therefore proposed that enhanced maternal care mediates the early handling effects compared to both nonhandling and maternal separation (Francis \& Meaney, 1999; Meaney, 2001). However, although it has been claimed that maternal separation deprives pups of maternal care (e.g. Caldji et al., 2000b) this has not been systematically studied. Present evidence suggests that maternal separation induces a compensatory bout of maternal care following reunion of dams and pups (Lonstein et al., 1998; Pryce et al., 2001; Zimmerberg et al., 2003), with no effect on maternal care throughout the rest of the day (Boccia \& Pedersen, 2001; Pryce et al., 2001). However, present data do not allow testing of the prediction made by Meaney (2001) that variations in maternal care can account for the effects of the different postnatal manipulations on offspring phenotype.

Here we therefore assessed the effects of early handling, maternal separation and nonhandling on maternal behaviour as well as the adult 
offspring's HPA and fear responses, with the aim of testing whether variations in maternal care can indeed explain the effects of these different neonatal manipulations on offspring phenotype.

\section{Materials and methods}

\section{Subjects}

Thirty-two pregnant Lister-hooded dams (Animal Services, Swiss Federal Institute of Technology, Zürich, Schwerzenbach) were housed in standard polycarbonate cages $(59.0 \times 38.5 \times 20.0 \mathrm{~cm})$ with sawdust bedding and ad libitum water and rodent pellets (Universal feed 3430, Moulin Kilba SA, Kaiseraugst, CH). They were maintained on a reversed $12: 12 \mathrm{~h}$ light : dark cycle (lights on at $19.00 \mathrm{~h}$ ) with temperature at $21 \pm 1{ }^{\circ} \mathrm{C}$ and relative humidity of $55 \pm 5 \%$. Prior to giving birth, they were randomly allocated to four treatment groups ( $n=8$ per group): maternal separation (MS), early handling of dams (EHd), early handling of dams and pups (EHdp), and non-handling (NH). Females were inspected daily at $14.00 \mathrm{~h}$ for delivery and day of birth was designated as postnatal day 0 (PND 0). One NH dam did not give birth to a viable litter. At PND 21, all offspring were weaned into same-gender groups of four littermates. One week prior to testing, all offspring were re-housed in pairs. All experimental manipulations were conducted under experimental permit in accordance with the Swiss Animal Protection Act (1978).

\section{Treatments}

From PND 1-13, dams and pups were exposed to the following postnatal manipulations: MS, placing dam in adjacent cage for $4 \mathrm{~h}$; Ehdp, placing both dam and pups separately in adjacent cages for $15 \mathrm{~min}$; Ehd, placing dam in adjacent cage for $15 \mathrm{~min}$; $\mathrm{NH}$, leaving dam and pups completely undisturbed (including no cage-cleaning). MS was initiated at $10.00 \mathrm{~h}$ and $\mathrm{EH}$ at $13.45 \mathrm{~h}$, such that all postnatal treatments ended at $14.00 \mathrm{~h}$. The EHdp and EHd treatments were used for the following reasons: postnatal manipulations traditionally involve placing the pups in separate cages, or placing both dams and pups separately in different cages (Pryce \& Feldon, 2003). In view of planned future studies on the effects of natural variations in nest attendance, we were interested in leaving the entire litter in the home cage and instead placing the dams in adjacent holding cages (EHd). However, to control for differences between this and traditional treatments, we also used a second early handling treatment for comparison in which both dams and pups were placed separately in adjacent cages (EHdp).

\section{Maternal behaviour}

Throughout PND 1-13, all cages were continuously video recorded for behavioural scoring. Small black-and-white cameras (one for each cage) with adjustable lenses (CSB-465CIR; Pacific Corporation, Tokyo, Japan) were mounted on a standard laboratory rack so as to face the short side of the cages. Twelve cameras each were linked to switcher boards (VU-08-BM, CES, Dübendorf, Switzerland) connected to time-lapse video recorders (Panasonic VCR AG-6740, Vitec, Dübendorf, Switzerland) and the switchers were set to allow for 15 -s observations per cage every $3 \mathrm{~min}$. Maternal behaviour was scored blindly at 3-min intervals for $1 \mathrm{~h}$ (20 samples/h) every third hour, starting at $14.00 \mathrm{~h}$, immediately following postnatal manipulations. Thus, for each dam a total of 160 samples per day $(8 \times 20)$ were obtained, except for MS dams, for which $1 \mathrm{~h}$ was missing due to the daily 4-h separation.

The following behaviours were scored (cf. e.g. Stern, 1996).

\section{Licking}

The dam hovers over the pups and holds one pup in her paws, performing repetitive licking movements, proceeding from the pup's head to trunk to anogenital region, referred to as 'licking/grooming' by Meaney (see e.g. Champagne et al., 2003).

\section{High kyphosis}

The dam is immobile in high upright dorsal arch posture supported by rigid fore- and hind-limbs, the head is depressed, the trunks and limbs are bilaterally symmetrical, and pups are attached to the nipples (part of Meaney's 'arched-back nursing').

\section{Low/partial kyphosis}

The dam is over the pups, relatively immobile, bilaterally symmetrical, with the head not depressed and in low dorsal arch posture supported by rigid fore- and hind-limbs (low kyphosis), or in low dorsal arch posture supported by rigid fore-limbs or rigid hind-limbs (referred to as 'partial kyphosis', part of 'arched-back nursing' by Meaney).

\section{Prone nursing}

The dam lies flat on top of the pups with little or no limb support (Meaney's 'blanket nursing').

\section{Supine nursing}

The dam lies on her side or back with some of the pups attached to the nipples (Meaney's 'passive nursing').

\section{Self-maintenance}

The dam is not in any form of physical contact with her pups. This behavioural category comprised feeding and drinking, active behaviour outside the nest, resting and self-grooming (licking, scratching and washing of the head and body) (Meaney's 'off the nest' or 'no contact').

\section{Undetectable}

This was scored when poor visibility prevented unambiguous identification of the behaviour. Overall, this accounted for $3.2 \%$ of all observations.

Based on Liu et al. (1997) and Champagne et al. (2003), we combined our categories licking, high kyphosis and low/partial kyphosis into a single behavioural category called 'active nursing' for further analyses [equivalent to Meaney's licking/grooming-archedback nursing (LG-ABN)].

\section{Novelty-induced suppression of drinking (NISD)}

This test of anxiety was developed and pharmacologically validated by Stout \& Weiss (1994) and is similar to the novelty-induced suppression of feeding test (e.g. Liu et al., 1997). From each litter, two males and two females were used. Starting at 11 weeks of age, rats were given restricted access to water for $2 \mathrm{~h}$ daily for 7 days prior to testing to guarantee high drinking motivation at the time of testing. Testing was conducted in a brightly lit room. One of two littermates was removed from the home cage and placed in a square open field for $10 \mathrm{~min}$. The open field was made of dark grey PVC $(80 \times 80 \times 60 \mathrm{~cm}$ high), with a water bottle hanging in the centre $14 \mathrm{~cm}$ above the floor. 
To control for motivational differences, the cage mate was left in the home cage and given normal access to water there. Behaviour was video-recorded and scored blindly by video observation. The following behaviours were scored: latency to start drinking (s), total time spent drinking (s) and time spent in the centre of the open field. After each session, the open field was thoroughly cleaned with an alcoholwater solution.

\section{Plasma ACTH and corticosterone response to restraint stress}

At the age of 11 weeks, from each litter one male and one female were taken from the home cage, carried by a familiar experimenter to an adjacent room and bled from the tail [time zero $\left(t_{0}\right) ; 0.2-0.4 \mathrm{~mL}$ collected into prechilled ethylenediamine tetraacetic acid (EDTA)coated tubes; Microvette; Sarstedt, Sevelen, Switzerland] by tail incision (Fluttert et al., 2000) within 2 min from entering the colony room, to obtain a blood sample for analysis of basal plasma ACTH and corticosterone. Subsequently, the rats were placed in a transparent Plexiglas restraint tube $(5 \mathrm{~cm}$ in diameter) of adjustable length for $25 \mathrm{~min}$ to induce a stress response. After $25 \mathrm{~min}$, they were bled from the same tail incision for a second time $\left(t_{25}\right)$ to obtain blood samples for analysis of the peak stress response before release from restraint and transport back to the colony room. At times $t_{60}$ and $t_{120}$, rats were again transported to the adjacent room, bled from the same tail incision for a third and fourth time to obtain blood samples for analysis of recovery from the stressor, and returned to the colony room. Samples were cool-centrifuged and the plasma stored at $-80{ }^{\circ} \mathrm{C}$ until assayed.

\section{Plasma corticosterone radioimmunoassay}

Plasma immunoreactive corticosterone titres were determined with an in-house ${ }^{3} \mathrm{H}$ radioimmunoassay validated for rat EDTA plasma. Diluted plasma samples were heated in a water bath at $90{ }^{\circ} \mathrm{C}$ for binding protein denaturation, and rabbit anticorticosterone serum (07-120016, ICN Biomedicals, Costa Mesa, CA, USA) was incubated with $\left[1,2,6,7-{ }^{3} \mathrm{H}\right]$ corticosterone (TRK 406, Amersham Switzerland, Zürich) and either corticosterone standard (Sigma, C-2505; 12.5$250 \mu \mathrm{L}$ per tube, in duplicate) or sample $(250 \mu \mathrm{L}$ at $1: 400$ dilution in duplicate) separation was achieved with dextran-coated charcoal.

\section{Plasma ACTH radioimmunoassay}

Plasma immunoreactive ACTH titres were quantified using an ACTH ${ }^{125}$ I radioimmunoassay kit for the determination of human ACTH in EDTA plasma (DiaSorin, Stillwater, MN, USA). To increase assay sensitivity and reduce the volume of plasma needed per measurement, the supplied assay protocol was slightly modified. ${ }^{125} \mathrm{I}$ tracer $(50 \mu \mathrm{L})$ and $50 \mu \mathrm{L}$ of antiserum were added to $150-\mu \mathrm{L}$ aliquots of the five standards (diluted 1:6 in distilled water to give concentrations of 4-120 pg/mL), to $150-\mu \mathrm{L}$ aliquots of two controls (diluted $1: 6$ in distilled water) and to $150 \mu \mathrm{L}$ of sample (diluted 1: 10 in distilled water). Standards and controls were measured in duplicate, samples singly in borosilicate glass tubes. Tubes were vortexed and incubated at $4{ }^{\circ} \mathrm{C}$ for $20 \mathrm{~h}$. Separation was achieved by adding precipitating complex $(250 \mu \mathrm{L})$, diluted $1: 2$ in distilled water, to all tubes except total count. Following brief vortexation and 20-min incubation at $20-25{ }^{\circ} \mathrm{C}$, tubes were centrifuged at $1500 \mathrm{~g}$ for $20 \mathrm{~min}$. The supernatant was aspirated and samples measured in a gamma scintillation counter (Minaxi (C); Packard, Downers Grove, IL, USA), 3-min count per tube. The ${ }^{125} \mathrm{I}$ radioimmunoassay was validated for ACTH in rat EDTA plasma. Inter-assay precision was $7.6 \%$ at $72-85 \%$ binding $(n=6)$ and $12.2 \%$ at $44-60 \%$ binding $(n=6)$, and intra-assay precision was $9.3 \%$ at $33-42 \%$ binding $(n=6)$. Assay sensitivity was $0.2 \mathrm{pg} /$ tube at $95 \%$ binding.

\section{Statistical analysis}

Data were analysed by repeated-measures ANOVA for split-plot designs. For the analysis of maternal behaviour the general model was 13 days $\times 7 \mathrm{~h} \times 4$ treatments. Treatment was a between-litter factor while all other variables were within-litter factors. Data on maternal behaviour were transformed to arcsine of the square root of the relative frequencies of behavioural scores. For reasons of clarity, all figures are based on nontransformed values. For analysis of time spent in the centre of the open field in the NISD test, the general model was 2 genders $\times 3$ treatments, while the number of animals drinking during the test session was analysed using the $\chi^{2}$ test. The general model for analysis of plasma levels of corticosterone and ACTH was 4 time points $\times 4$ treatments $\times 2$ genders. Treatment was a betweenlitter factor while gender and time points were within-litter factors. Fisher PLSD post hoc tests were used where appropriate.

\section{Results \\ Early handling, maternal care and HPA response to stress}

To examine whether effects of early handling depend upon manipulating both pups and dams (EHdp) or are the same if only the dams are removed (EHd), the effects of these two treatments on maternal behaviour and the adult offspring's HPA and fear responses were analysed. Repeated-measures ANOVA with treatment as a between-subjects factor and observation day (PND 1-13) as a within-subjects factor revealed no significant effects on active nursing $\left(F_{1,14}=0.6\right.$, NS), passive nursing $\left(F_{1,14}=0.8\right.$, NS) or self-maintenance behaviours $\left(F_{1,14}=0.4, \mathrm{NS}\right)$, and there were no significant interactions between these three behavioural categories and observation day $\left(F_{12,168}=0.4, \quad \mathrm{NS} ; \quad F_{12,168}=1.5, \quad \mathrm{NS}\right.$; $F_{12,168}=0.9$, NS, respectively). Thus, both overall levels of maternal behaviours and changes across the postnatal period did not differ between the two treatments. In line with this, adult offspring of the two treatment groups differed in neither basal ACTH $\left(F_{1,14}=0.3\right.$, NS) nor corticosterone levels $\left(F_{1,14}=0.2, \mathrm{NS}\right)$, nor in their ACTH $\left(F_{1,14}=0.01, \mathrm{NS}\right)$ and corticosterone responses $\left(F_{1,14}=1.7\right.$, NS) to $25 \mathrm{~min}$ restraint stress. Furthermore, neither the number of animals drinking in the novel open field arena $\left(\chi^{2}=1.0\right.$, NS) nor the time spent in the centre of the arena $\left(F_{1,61}=1.0, \mathrm{NS}\right)$ differed between EHd and EHdp animals. Based on these results, the two early handling treatments EHdp and EHd were pooled (further called $\mathrm{EH}$ ) for all further analyses.

\section{Postnatal manipulations and maternal behaviour}

Table 1 presents the average scores for the whole observation period for all behaviours that were recorded. For further analyses, licking, high kyphosis and low/partial kyphosis were combined into a single category of active nursing which is consistent with the category LG-ABN in the work of Meaney's group (e.g. Champagne et al., 2003; see also Materials and methods).

Figure 1 depicts mean daily levels of active nursing from PND 1-13 for NH, EH and MS. Active nursing (Fig. 1a) gradually decreased across days (days, $F_{12,336}=13.4, P<0.01$ ). However, neonatal manipulations significantly affected levels of active nursing (treatment, $F_{2,28}=6.5, P<0.01$ ). Both $\mathrm{EH}$ and $\mathrm{MS}$ dams showed 
TABLE 1. Average durations of the different maternal behaviours

\begin{tabular}{|c|c|c|c|c|c|c|c|}
\hline \multirow[b]{2}{*}{ Treatment } & \multicolumn{7}{|c|}{ Average duration (h) } \\
\hline & Licking & $\begin{array}{l}\text { High } \\
\text { kyphosis }\end{array}$ & $\begin{array}{l}\text { Low/partial } \\
\text { kyphosis }\end{array}$ & $\begin{array}{l}\text { Active } \\
\text { nursing }\end{array}$ & $\begin{array}{l}\text { Prone } \\
\text { nursing }\end{array}$ & $\begin{array}{l}\text { Supine } \\
\text { nursing }\end{array}$ & $\begin{array}{l}\text { Self- } \\
\text { maintenance }\end{array}$ \\
\hline $\mathrm{NH}$ & $3.2 \pm 0.1$ & $1.1 \pm 0.1$ & $3.9 \pm 0.1$ & $8.2 \pm 0.3$ & $1.7 \pm 0.1$ & $1.7 \pm 0.1$ & $7.6 \pm 0.2$ \\
\hline $\mathrm{EH}$ & $3.9 \pm 0.1$ & $1.1 \pm 0.1$ & $4.7 \pm 0.1$ & $9.7 \pm 0.2$ & $1.7 \pm 0.1$ & $1.4 \pm 0.1$ & $6.2 \pm 0.1$ \\
\hline MS & $4.6 \pm 0.1$ & $1.3 \pm 0.1$ & $4.8 \pm 0.1$ & $10.7 \pm 0.3$ & $1.6 \pm 0.1$ & $1.5 \pm 0.1$ & $5.1 \pm 0.1$ \\
\hline
\end{tabular}

Data are presented as averages $( \pm$ SEM). Daily means per hour were averaged over the entire observation period. Included is the combined category of active nursing (licking, high kyphosis, low/partial kyphosis) which was used for all further analyses. Active nursing is equivalent to LG-ABN used by Meaney and colleagues (e.g. Champagne et al., 2003).

(a)

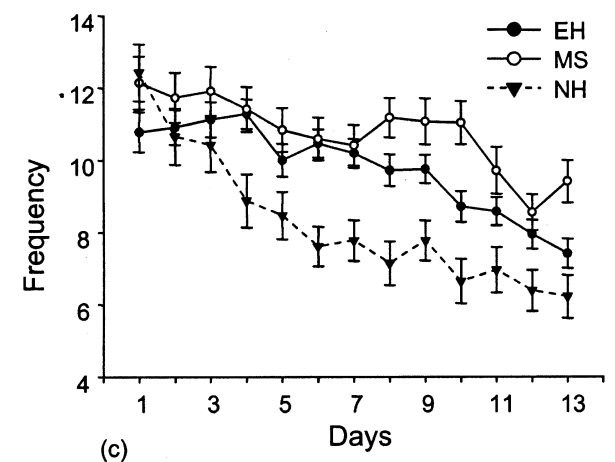

(c)

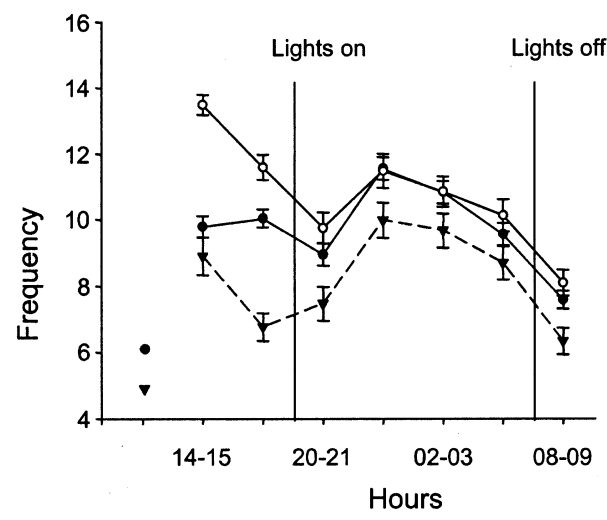

(b)

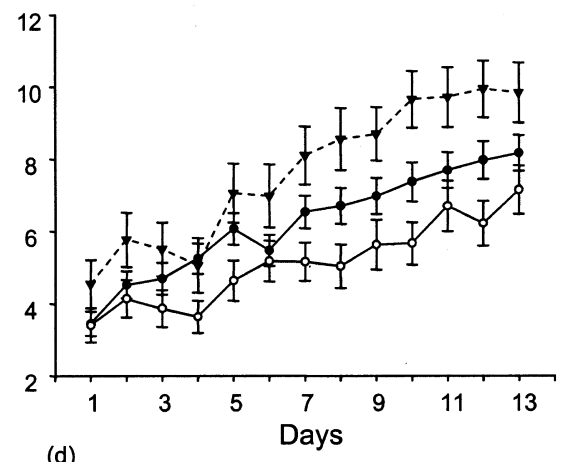

(d)

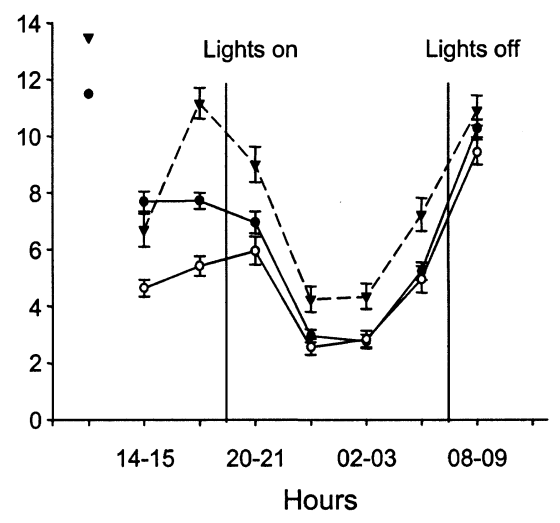

FIG. 1. Effects of postnatal manipulations on maternal behaviour. (a,b) Daily frequency (mean $/ \mathrm{h} \pm \mathrm{SEM}$ ) of (a) active nursing and (b) self-maintenance behaviours shown by dams subjected daily to early handling ( $\mathrm{EH} ; n=16)$, maternal separation (MS, $n=8)$ or nonhandling $(\mathrm{NH}, n=7)$. Daily scores are based on seven daily 1-h sampling sessions. (c,d) Diurnal pattern of (c) active nursing and (d) self-maintenance scores (mean \pm SEM) shown by EH $(n=16)$, MS $(n=8)$ and $\mathrm{NH}$ dams $(n=7)$. Scores from each of seven (MS) or eight (EH, $\mathrm{NH}$ ) daily 1-h sampling sessions were averaged across PND 1-13. MS dams were separated from the pups from 10.00 to $1400 \mathrm{~h}$, while EH dams were separated from the pups from 13.45 to $14.00 \mathrm{~h}$. Reunion occurred in both groups at $14.00 \mathrm{~h}$. Isolated data points indicate average levels of (c) active nursing and (d) self-maintenance in $\mathrm{NH}$ and $\mathrm{EH}$ dams from 11.00 to $12.00 \mathrm{~h}$, when MS dams were separated from their pups for maternal separation (hence no data for MS dams).

significantly higher levels of active nursing than $\mathrm{NH}$ dams (post hoc tests, EH vs. NH $P<0.05$, MS vs. NH $P<0.05)$ and MS dams tended to score even higher than EH dams (MS vs. EH $P=0.1$ ). This difference between $\mathrm{NH}$ and the two other treatments emerged on PND 4 and remained stable throughout the remaining observation period. Looking into the diurnal pattern (Fig. 1c) revealed that active nursing was elevated throughout the day in EH and MS dams compared to $\mathrm{NH}$ dams. However, MS dams showed significantly enhanced levels of active nursing compared to both $\mathrm{EH}$ and $\mathrm{NH}$ dams in the hours following reunion of dams and pups $(P<0.01)$, while they did not differ from $\mathrm{EH}$ dams for the remaining of the day (although both showed higher levels than $\mathrm{NH}$ ).
Differences in active nursing were mirrored in the expression of self-maintenance behaviours (Fig. $1 \mathrm{~b}$ and d). Self-maintenance gradually increased over lactation days (days, $F_{12,336}=27.4$, $P<0.001)$ and was significantly affected by neonatal manipulations (treatment, $F_{2,28}=16.7, P<0.01$ ). Both $\mathrm{EH}$ and MS dams scored significantly lower than $\mathrm{NH}$ dams $(P<0.01)$, and MS dams also scored lower than EH dams $(P<0.01)$. These differences were stable throughout the observation period. Looking at the diurnal pattern (Fig. 1d), however, the overall difference between MS and EH dams was again based on the daily period following reunion of dam and pups, while they did not differ during the remaining of the day. By contrast, both $\mathrm{EH}$ and MS dams scored lower than $\mathrm{NH}$ dams 
(a)

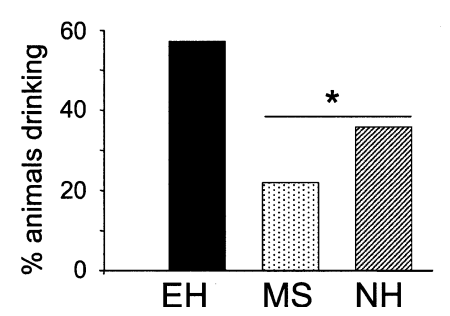

(b)

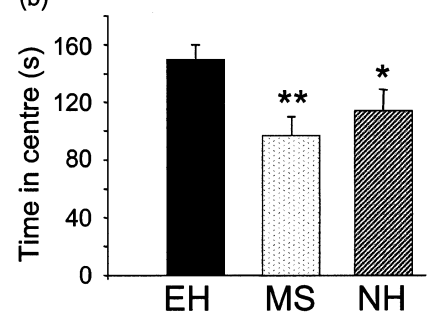

FIG. 2. Effects of postnatal manipulations on behavioural fearfulness. (a) Proportion (\%) of animals subjected to early handling (EH, $n=64)$, maternal separation (MS, $n=32$ ) and nonhandling (NH, $n=27)$ drinking within a 10-min test session from a drinker presented in a novel arena. (b) Mean ( \pm SEM) time spent in the centre of the novel arena by EH, MS and $\mathrm{NH}$ animals during the 10 -min test session. In the absence of any significant gender effect, data of males and females were pooled. ${ }^{*} P<0.01,{ }^{*} P<0.05$; multiple comparisons $\mathrm{EH}$ vs. MS and $\mathrm{NH}$.

throughout the day, except for the early dark period when all groups showed maximal levels, and the period following reunion of dam and pups when $\mathrm{EH}$ dams did not differ from $\mathrm{NH}$ dams.

\section{Effects on fearfulness in adult offspring}

When given access to water in the home cage, all rats immediately started drinking (data not shown), whereas access to water in a novel open field suppressed drinking dramatically, preventing many rats from drinking within the time of the test session. Instead of the latency to drink and the time spent drinking, we therefore analysed treatment effects on the number of animals drinking. Significantly more EH offspring (57.1\%) than MS $(21.8 \%)$ or $\mathrm{NH}$ offspring $(35.7 \%)$ approached the drinker in the centre of the arena $\left(\chi^{2}=7.2\right.$, $P<0.05$; Fig. 2a), whereas MS and NH animals did not differ $\left(\chi^{2}=1.0, \mathrm{NS}\right)$. Moreover, EH offspring spent significantly more time in the centre of the arena than MS and $\mathrm{NH}$ offspring (treatment, $F_{2,119}=5.4, P<0.01$; Fig. 2 b). Neither the number of animals drinking in the open field arena $\left(\chi^{2}=0.9\right.$, NS) nor the time spent in the centre of the open field $\left(F_{1,16}=0.9, \mathrm{NS}\right)$ were affected by gender.

\section{Effects on HPA responses in adult offspring}

Physical restraint induced massive increases in plasma ACTH and corticosterone levels which were, however, back to basal levels by t120 (time points, $F_{3,84}=55.1, P<0.001$ ). Postnatal manipulations had no effect on basal levels of ACTH $\left(F_{2,28}=0.1\right.$, NS) or corticosterone $\left(F_{2,28}=0.3\right.$, NS), but significantly affected peak levels of response to restraint (Fig. 3a and b). Thus, peak levels of ACTH did not differ between MS and $\mathrm{NH}$ offspring, but were significantly elevated in these two groups compared to EH offspring (treatment, $\left.F_{2,28}=4.9, P<0.05\right)$. These results were paralleled by the corticosterone responses to restraint stress. Again, MS and $\mathrm{NH}$ offspring did not differ, but showed significantly higher peak levels than EH offspring. Although females had higher ACTH and corticosterone levels than males (gender main effect on ACTH, $F_{1,28}=18.8$, $P<0.01$, and corticosterone, $\left.F_{1,28}=70.8, P<0.01\right)$, there were no significant gender $\times$ treatment interactions $\left(F_{2,28}=0.23\right.$, NS and $F_{2,28}=0.1$, NS, respectively), indicating that treatment effects were independent of gender. Consequently, males and females were pooled for Fig. 3. Because of this pooling of the two genders, and because blood samples were taken during the middle of the dark phase when HPA activity is still elevated compared to the early light phase
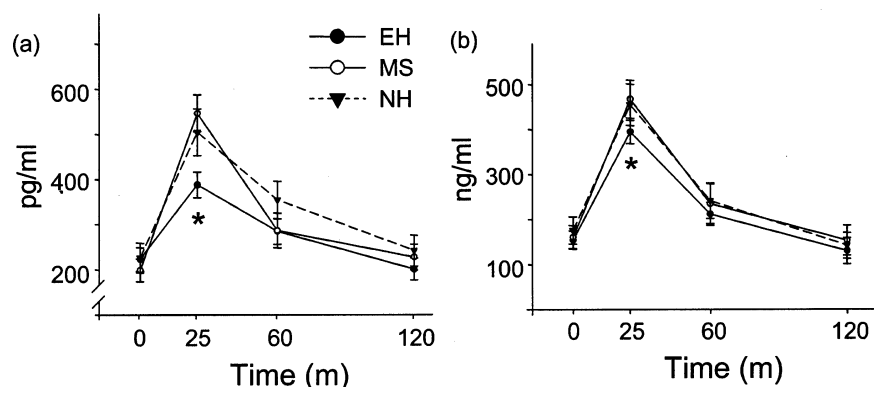

FIG. 3. Effects of postnatal manipulations on HPA responses. Basal levels $\left(t_{0}\right)$, peak response $\left(t_{25}\right)$ and recovery $\left(t_{60}, t_{120}\right)$ of (a) plasma ACTH and (b) plasma corticosterone levels shown by animals subjected to early handling $(\mathrm{EH}, n=32)$, maternal separation $(\mathrm{MS}=16)$ and nonhandling $(\mathrm{NH}=14)$ in response to 25 -min restraint stress. In the absence of any significant treatment $\times$ gender interaction, data of males and females were pooled. ${ }^{*} P<0.05$; post hoc tests EH vs. MS and NH.

(Nolan et al., 2000), basal levels of ACTH and corticosterone appear relatively high, but lie within the normal range of diurnal variation (e.g. van Haarst et al., 1996). Similarly, peak response levels lie within the range published for this strain (e.g. Manahan-Vaughan \& Braunewell, 1999), although they appear relatively high compared to data obtained from other strains. Fluttert et al. (2000) have shown that repeated blood sampling by this method of tail incision does not affect corticosterone levels, and levels of corticosterone taken from the tail incision and from the jugular vein (in cannulated rats) were perfectly correlated.

\section{Discussion}

Our findings demonstrate dissociation in the effects of different neonatal maternal separations on maternal care and the adult offspring's stress and fear responses. Both EH and MS induced a more active nursing style and elevated levels of maternal care compared to nonhandled controls $(\mathrm{NH})$. In terms of total maternal care these two treatments were indistinguishable, the only difference between them being the temporal distribution of maternal care. In the adult offspring, however, EH, but not MS, resulted in reduced HPA responses to physical restraint and reduced fear responses towards novelty compared to $\mathrm{NH}$.

To examine whether the behavioural scoring used here was consistent with that of Meaney and colleagues, we compared the relative proportions of the different behavioural categories recorded in NH dams with similar data obtained from a random population of 115 nonhandled dams as recently published by Champagne et al. (2003). Table 2 shows that the relative proportions of the different maternal

TABLE 2. Maternal behaviour in $\mathrm{NH}$ dams: comparison of the present study with that of Champagne et al., 2003*

\begin{tabular}{llc}
\hline & \multicolumn{2}{c}{ Maternal behaviour $(\%)$} \\
\cline { 2 - 3 } Behaviour & This study & Champagne et al. $(2003)^{*}$ \\
\hline Active nursing (LG-ABN) & 43 & 48 \\
Blanket nursing & 9 & 6 \\
Passive nursing & 9 & 9 \\
Self-maintenance (no contact) & 39 & 37 \\
\hline
\end{tabular}

*Data from a population of 115 nonhandled dams. 
behaviours are virtually identical in these two studies, indicating that behavioural scoring was fully consistent. Taken together, our findings demonstrate that the effects of different neonatal maternal separations on the adult offspring's stress and fear responses cannot be fully explained by the effects of these manipulations on the amount of maternal care.

\section{Postnatal manipulations and fear and stress in the adult offspring}

Both plasma ACTH and corticosterone levels after 20 min restraint stress were reduced in EH compared to NH offspring. Furthermore, $\mathrm{NH}$ offspring spent less time than $\mathrm{EH}$ offspring in the centre of a novel arena, and fewer $\mathrm{NH}$ offspring than $\mathrm{EH}$ offspring approached a drinker presented in the novel arena. The latter effect cannot be explained by motivational differences because both groups immediately started drinking when the drinker was presented in the home cage. These results confirm previous research showing that early handling attenuates HPA responses and behavioural fearfulness in the adult offspring compared to nonhandling (recent reviews by Meaney, 2001; Pryce \& Feldon, 2003).

In contrast, maternal separation has been claimed to produce exactly opposite effects to early handling on the adult offspring's stress and fear responses (Francis \& Meaney, 1999; Meaney, 2001; see also Plotsky \& Meaney, 1993; Biagini et al., 1998; McCormick et al., 2002). Contrary to this view, MS offspring in the present study did not differ from NH offspring, either in HPA reactivity or in behavioural fearfulness. Although the possibility of a ceiling effect due to nearmaximal hormonal output cannot be ruled out completely, this is unlikely to account for the lack of differences in HPA reactivity between $\mathrm{NH}$ and MS offspring, for the following two reasons. First, if this were the case it should equally apply to EH offspring. However, peak response levels in EH were significantly reduced compared to both NH and MS in both hormones. In fact, differences in maximal output levels may be part of the treatment effects, although further research into the underlying physiological mechanisms will be needed to examine this hypothesis properly. Second, the behavioural test of fearfulness is likely to be less stressful than the 25-min tube restraint. However, the behavioural responses of all three treatment groups in this test were fully consistent with the hormonal measures from the restraint stress test (including the lack of a significant difference between NH and MS rats). Thus, our results resemble those of other authors who have failed to find enhancing effects of maternal separation on later stress responses compared with nonhandled controls (e.g. Plotsky \& Meaney, 1993; Caldji et al., 2000b; Liu et al., 2000).

Two reasons might explain why, contrary to empirical evidence, the view prevails that early handling and maternal separation produce opposite profiles compared to undisturbed controls. First, early handling is traditionally compared with undisturbed controls, whereas maternal separation is often compared with either early handling (cf. Matthews \& Robbins, 2003) or with standard husbandry controls (e.g. Ladd et al., 1996), thereby hampering interpretation of the effects (Lehmann \& Feldon, 2000). Secondly, maternal separation is thought to be stressful to both dam and pups and to deprive pups of maternal care (Meaney, 2001). Therefore, the idea that a treatment as severe as daily maternal separations for up to $6 \mathrm{~h}$ should produce the same effects as leaving dam and pups completely undisturbed may appear fundamentally counterintuitive. We therefore examined in detail the effects of these manipulations on maternal care, the proposed mediator of offspring phenotype.

\section{Early handling and maternal care}

Early handling normally involves removal of all animals from the home cage and placing the pups in separate containers for 3-15 min, while the dam is placed back in the home cage or left in a holding cage (Pryce \& Feldon, 2003). Consequently, the treatment is considered to act on the pups through their manipulation, separation from the dam, exposure to novelty, and sometimes isolation, yet the relative contribution of these different aspects of the treatment has remained elusive (Hall, 1998; Pryce \& Feldon, 2003).

Our findings clearly demonstrate that the effects of early handling are independent of pup handling per se. Both handling dams and pups (the classical treatment), and handling dams only while leaving pups untouched in the home cage, produced the same effects on maternal care and offspring phenotype. This suggests that mother-offspring separation per se could account for early handling effects. Alternatively, early handling could simply act by disturbing the safety and stability of the nest environment. In support of the latter explanation, standard husbandry routines (i.e. weekly cage changes) without mother-offspring separations appear to produce the same neurobehavioural profile as early handling (Caldji et al., 2000b; Pryce et al., 2001; Pryce \& Feldon, 2003). These results appear to contradict the recent findings by Tang and colleagues (Tang, 2001; Tang et al., 2003), showing that pups that were daily exposed to novelty for brief periods differed in basal corticosterone levels, brain asymmetry and memory performance from littermates that were left in the home cage. However, the fact that basal corticosterone levels are not normally affected by other postnatal treatments (e.g. Meaney et al., 1989; Plotsky \& Meaney, 1993; Viau et al., 1993; Liu et al., 2000; present study) suggests that the within-litter effects of Tang's split-litter treatment and our between-litter effects of the two early handling treatments are not necessarily comparable.

\section{Postnatal manipulations and maternal style}

In contrast to maternal separation, in which the 3-6-h mother-infant separations clearly exceed the normal intervals between nursing bouts, early handling is not considered to constitute maternal deprivation (Liu et al., 1997; Pryce et al., 2001). Our results provide further support for this view. Although EH increased active nursing compared to $\mathrm{NH}$, effect size remained largely constant throughout the day, with no compensatory nursing in the hours following reunion. Only between 17.00 and $18.00 \mathrm{~h}$ was the elevation in $\mathrm{EH}$ compared to $\mathrm{NH}$ higher than at other time points. However, this may reflect the general up-regulation in maternal care rather than compensation, given that this occurred 3-4 $\mathrm{h}$ after reunion and no compensation occurred in the hour following reunion. MS dams, in contrast, showed significantly elevated levels of active nursing compared to both $\mathrm{NH}$ and $\mathrm{EH}$ dams following reunion. This compensatory bout of active nursing in MS dams confirms earlier reports (Lonstein et al., 1998; Pryce et al., 2001; Zimmerberg et al., 2003) and indicates that, despite equal overall levels of active nursing, there were substantial qualitative differences in the maternal care received by EH and MS pups as well as between $\mathrm{NH}$ and MS pups.

Paradoxically, NH and MS offspring did not differ despite differences in total maternal care while EH and MS offspring did differ, despite indistinguishable levels of maternal care. Furthermore, levels of active nursing did not correlate with later stress responsiveness or fearfulness, in any group (data not shown). This dissociation between the effects on maternal care and the effects on offspring phenotype indicates that the total amount of maternal care cannot be the sole factor. Therefore, other aspects of maternal care (e.g. temporal 
distribution), or another factor entirely, must contribute to the effects of EH and MS on offspring phenotype.

\section{Adaptive and pathological effects of postnatal manipulations}

How could it be that maternal separation might affect offspring phenotype in the same way as leaving dam and pups completely undisturbed? According to Francis \& Meaney (1999), increasing environmental demands placed on the dams interfere with maternal care, which in turn induces higher fear and stress responses in the offspring (see also Meaney, 2001). However, because handled rather than nonhandled dams are exposed to a more challenging environment (the daily handling procedure), this explanation is somewhat inconsistent with the evidence. Furthermore, it fails to explain how the undisturbed condition of nonhandling and the adverse condition of maternal separation could both produce similar effects.

One possible explanation would be that nonhandling and maternal separation produce similar phenotypes, but for different reasons. For example, the difference between $\mathrm{EH}$ and $\mathrm{NH}$ might indeed be mediated by variations in maternal care as proposed by Francis \& Meaney (1999), whereby a more active maternal style could serve to down-regulate the offspring's stress system. By contrast, the high stress reactivity and fearfulness of MS offspring might reflect the maladaptive (i.e. pathological) outcome of a disruption of pup homeostasis, mediated by repeated prolonged maternal separations (cf. Hofer, 1994a,b; Kuhn \& Schanberg, 1998). Despite receiving the same active maternal style as EH pups, and compensatory maternal care following separations, MS offspring develop high stress reactivity. Thus, the disruption of normal intervals between nursing bouts (induced by prolonged dam-pup separation) and enhanced maternal care (induced by the manipulation) could represent two independent yet opposing factors in the mediation of offspring phenotype. This could also explain the conflicting findings from maternal separation studies: depending on the exact protocol, one or the other factor might come to prevail. Furthermore, MS and $\mathrm{NH}$ might produce similar outcomes in terms of HPA responses and behavioural measures of fearfulness despite underlying neural differences. Francis et al. (2002) have shown that environmental enrichment during the peripubertal period completely reverses the effects of MS compared to EH on both HPA and behavioural responses to stress, with no effect on CRF mRNA expression. This is suggestive of a functional reversal through compensation for, rather than reversal of, the underlying neural effects of maternal separation (Francis et al., 2002). The nature of these compensatory mechanisms is currently unknown. Thus, further studies into the neural changes associated with different postnatal manipulations will have to reveal the exact pathways by which each treatment mediates its effects on HPA responses.

The implications of the present results are twofold. First, they suggest a re-examining of the maternal mediation hypothesis (Smotherman \& Bell, 1980; Meaney, 2001) concerning the effects of early postnatal manipulations on the development of stress and fear responses. Second, they suggest that at least two independent factors contribute to these effects. Besides the amount of maternal care, other possible factors could be the temporal distribution of nursing bouts or the duration of mother-offspring separation. Identifying these factors and their specific effect on the HPA system could have important implications for our understanding of the relationships between the early mother-infant relationship and the development of stress disorders in animals and man.

\section{Acknowledgements}

This study was supported by the ETH Zürich. Many thanks to Wolfgang Langhans for hosting the study, to the Technical Service at the ETH laboratory in Schwerzenbach, and to Myrtha Arnold and Toni Moses for their help with the lab work.

\section{Abbreviations}

EDTA, ethylenediamine tetraacetic acid; EH, early handling; EHd, early handling of dams; EHdp, early handling of dams and pups; HPA, hypothalamic-pituitary-adrenocortical; LG-ABN, licking/grooming-arched-back nursing; MS, maternal separation; NH, non-handling; NISD, novelty-induced suppression of drinking; PND, postnatal day.

\section{References}

Ader, R. \& Grota. L.J. (1969) Effects of early experience on adrenocortical reactivity. Physiol. Behav., 4, 303-305.

Bhatnagar, S., Shanks, N. \& Meaney, M.J. (1995) Hypothalamic-pituitaryadrenal function in handled and nonhandled rats in response to chronic stress. J. Neuroendocrinol., 7, 107-119.

Biagini, G., Pich, E.M., Carani, C., Marrama, P. \& Agnati, L.F. (1998) Postnatal maternal separation during the stress hyporesponsive period enhances the adrenocortical response to novelty in adult rats by affecting feedback regulation in the CA1 hippocampal field. Int. J. Dev. Neurosci., 16, 187-197.

Boccia, M.L. \& Pedersen, C.A. (2001) Brief vs. long maternal separations in infancy: contrasting relationships with adult maternal behaviour and lactation levels of aggression and anxiety. Psychoneuroendocrinology, 26, 657-672.

Bodnoff, S.R., Suranyi-Cadotte, B., Quirion, R. \& Meaney, M.J. (1987) Postnatal handling reduces novelty-induced fear and increases $\left[{ }^{3} \mathrm{H}\right]$ flunitrazepam binding in rat brain. Eur. J. Pharmacol., 144, 105-107.

Caldji, C., Diorio, J. \& Meaney, M.J. (2000a) Variations in maternal care in infancy regulate the development of stress reactivity. Biol. Psychiatry, 48, 1164-1174.

Caldji, C., Francis, D., Sharma, S., Plotsky, P.M. \& Meaney, M.J. (2000b) The effects of early rearing environment on the development of GABAA and central benzodiazepine receptor levels and novelty-induced fearfulness in the rat. Neuropsychopharmacology, 22, 219-229.

Caldji, C., Tannenbaum, B., Sharma, S., Francis, D., Plotsky, P.M. \& Meaney, M.J. (1998) Maternal care during infancy regulates the development of neural systems mediating the expression of fearfulness in the rat. Proc. Natl Acad. Sci. USA, 95, 5335-5340.

Champagne, F.A., Francis, D.D., Mar., A. \& Meaney, M.J. (2003) Variations in maternal care in the rat as a mediating influence for the effects of environment on development. Physiol. Behav., 79, 359-371.

Denenberg, V.H. (1964) Critical periods, stimulus input, and emotional reactivity: a theory of infantile stimulation. Psychol. Rev., 71, 335-351.

Fluttert, M., Dalm, S. \& Oitzl, M.S. (2000) A refined method for sequential blood sampling by tail incision in rats. Laboratory Anim., 34, 372-378.

Francis, D.D. \& Meaney, M.J. (1999) Maternal care and the development of stress responses. Curr. Opin. Neurobiol., 9, 128-134.

Francis, D.D., Diorio, J., Plotsky, P.M. \& Meaney, M.J. (2002) Environmental enrichment reverses the effects of maternal separation on stress reactivity. J. Neurosci., 22, 7840-7843.

van Haarst, A.D., Oitzl, M.S., Workel, J.O. \& de Kloet, E.R. (1996) Chronic brain glucocorticoid receptor blockade enhances the rise in circadian and stress-induced pituitary-adrenal activity. Endocrinology, 137, 4935-4943.

Hall, F.S. (1998) Social deprivation of neonatal, adolescent, and adult rats has distinct neurochemical and behavioural consequences. Crit. Rev. Neurobiol., 12, 129-162.

Hilakivi-Clarke, L.A., Turkka, J., Lister, R.G. \& Linnoila, M. (1991) Effects of early postnatal handling on brain beta-adrenoceptors and behaviour in tests related to stress. Brain. Res., 542, 286-292.

Hofer, M.A. (1994a) Early relationships as regulators of infant physiology and behaviour. Acta Paediatr., 397, S9-S18.

Hofer, M.A. (1994b) Hidden regulators in attachment, separation, and loss. [In Fox, N.A. (ed.), The Development of Emotion Regulation: Biological and Behavioural Considerations.] Monogr. Soc. Res. Child Dev., 59, 192-283.

Kuhn, C.M. \& Schanberg, S.M. (1998) Responses to maternal separation: mechanisms and mediators. Int. J. Dev. Neurosci., 16, 261-270.

Ladd, C.O., Owens, M.J. \& Nemeroff, C.B. (1996) Persistent changes in corticotropin-releasing factor neuronal systems induced by maternal deprivation. Endocrinology, 137, 1212-1218. 
Lehmann, J. \& Feldon, J. (2000) Long-term biobehavioural effects of maternal separation in the rat: consistent or confusing? Rev. Neurosci., 11, 383-408.

Levine, S. (1957) Infantile experience and resistance to physiological stress. Science, 126, 405.

Levine, S. (1962) Plasma-free corticosterone response to electric shock in rats stimulated in infancy. Science, 135, 795-796.

Levine, S., Haltmeyer, G.C., Karas, G.G. \& Denenberg, V.H. (1967) Physiological and behavioural effects of infantile stimulation. Physiol. Behav., 2, 55-59.

Liu, D., Diorio, J., Day, J.C., Francis, D.D. \& Meaney, M.J. (2000) Maternal care, hippocampal synaptogenesis and cognitive development in rats. Nat. Neurosci., 3, 799-806.

Liu, D., Diorio, J., Tannenbaum, B., Caldji, C., Francis, D., Freedman, A., Sharma, S., Pearson, D., Plotsky, P.M. \& Meaney, M.J. (1997) Maternal care, hippocampal glucocorticoid receptors, and hypothalamic-pituitary-adrenal responses to stress. Science, 277, 1659-1662.

Lonstein, J.S., Simmons, D.A., Swann, J.M. \& Stern, J.M. (1998) Forebrain expression of c-fos due to active maternal behaviour in lactating rats. Neuroscience, 82, 267-281.

Manahan-Vaughan, D. \& Braunewell, K.H. (1999) Novelty acquisition is associated with induction of hippocampal long-term depression. Proc. Natl Acad. Sci. USA, 96, 8739-8744.

Matthews, K. \& Robbins, T.W. (2003) Early experience as a determinant of adult behavioural responses to reward: the effects of repeated maternal separation in the rat. Neurosci. Biobehav. Rev., 27, 45-55.

McCormick, C.M., Kehoe, P., Mallinson, K., Cecchi, L. \& Frye, C.A. (2002) Neonatal isolation alters stress hormone and mesolimbic dopamine release in juvenile rats. Pharmacol. Biochem. Behav., 73, 77-85.

McIntosh, J., Anisman, H. \& Merali, Z. (1999) Short- and long-periods of neonatal maternal separation differentially affect anxiety and feeding in adult rats: gender-dependent effects. Brain. Res. Dev. Brain. Res., 113, 97-106.

Meaney, M.J. (2001) Maternal care, gene expression, and the transmission of individual differences in stress reactivity across generations. Annu. Rev. Neurosci., 24, 1161-1192.

Meaney, M.J., Aitken, D.H., Viau, V., Sharma, S. \& Sarrieau, A. (1989) Neonatal handling alters adrenocortical negative feedback sensitivity and hippocampal type II glucocorticoid receptor binding in the rat. Neuroendocrinology, 50, 597-604.
Nolan, L.A., Windle, R.J., Wood, S.A., Kershaw, Y.M., Lunness, H.R., Lightman, S.L., Ingram, C.D. \& Levy, A. (2000) Chronic iodine deprivation attenuates stress-induced and diurnal variation in corticosterone secretion in female wistar rats. J. Neuroendocrinol., 12, 1149-1159.

Ogawa, T., Mikuni, M., Kuroda, Y., Muneoka, K., Mori, K.J. \& Takahashi, K. (1994) Periodic maternal deprivation alters stress response in adult offspring: potentiates the negative feedback regulation of restraint stress-induced adrenocortical response and reduces the frequencies of open field-induced behaviours. Pharmacol. Biochem. Behav., 49, 961-967.

Plotsky, P.M. \& Meaney, M.J. (1993) Early, postnatal experience alters hypothalamic corticotropin-releasing factor (CRF) mRNA, median eminence CRF content and stress-induced release in adult rats. Brain. Res. Mol. Brain. Res., 18, 195-200.

Pryce, C.R., Bettschen, D. \& Feldon, J. (2001) Comparison of the effects of early handling and early deprivation on maternal care in the rat. Dev. Psychobiol., 38, 239-251.

Pryce, C.R. \& Feldon, J. (2003) Long-term neurobehavioural impact of the postnatal environment in rats: manipulations, effects and mediating mechanisms. Neurosci. Biobehav. Rev., 27, 57-71.

Smotherman, W.P. \& Bell, R.W. (1980) Maternal mediation of early experience. In Bell, R.W., Smotherman, W. \& P. (eds), Maternal influences and early behavior. Spectrum, New York, pp. 201-210.

Stern, J.M. (1996) Trigeminal lesions and maternal behaviour in Norway rats. II. Disruption of parturition. Physiol. Behav., 60, 187-190.

Stout, J.C. \& Weiss, J.M. (1994) An animal model for measuring behavioral responses to anxiogenic and anxiolytic manipulations. Pharmacol. Biochem. Behav., 47, 459-465.

Tang, A.C. (2001) Neonatal exposure to novel environment enhances hippocampal-dependent memory function during infancy and adulthood. Learn. Mem., 8, 257-264.

Tang, A.C., Reeb, B.C., Romeo, R.D. \& McEwen, B.S. (2003) Modification of social memory, hypothalamic-pituitary-adrenal axis, and brain asymmetry by neonatal novelty exposure. J. Neurosci., 23, 8254-8260.

Viau, V., Sharma, S., Plotsky, P.M. \& Meaney, M.J. (1993) Increased plasma $\mathrm{ACTH}$ responses to stress in nonhandled compared with handled rats require basal levels of corticosterone and are associated with increased levels of ACTH secretagogues in the median eminence. J. Neurosci., 13, 1097-1105

Zimmerberg, B., Rosenthal, A.J. \& Stark, A.C. (2003) Neonatal social isolation alters both maternal and pup behaviours in rats. Dev. Psychobiol., 42, 52-63. 